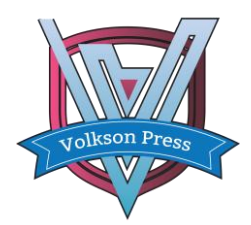

Contents List available at VOLKSON PRESS

Mechanical and Control Engineering (MCE)

DOI : http://doi.org/10.26480/wsmce.01.2017.95.98

\title{
METHOD FOR WEIGHT DECISION-MAKING OF WIRELESS NETWORK PERFORMANCE INDICATOR BASED ON ENTROPY METHOD
}

\author{
Zhang Ruifang ${ }^{*}$, Chen Yang2, Liu Yangxue ${ }^{1^{*}}$ \\ ${ }^{1}$ College of Mechanical and Control Engineering, Guilin University of Technology, Guilin, China \\ ${ }^{2}$ Network security detachment Guilin Municipal Public Security Bureau, Guilin, China \\ *Corresponding Author Email: 631600961@qq.com, joanna.liu.zhao@foxmail.com
}

This is an open access article distributed under the Creative Commons Attribution License, which permits unrestricted use, distribution, and reproduction in any medium, provided the original work is properly cited

\section{ARTICLE DETAILS}

\section{Article History:}

Received 02 october 2017 Accepted 06 october 2017 Available online 11 november 2017

Keywords

WLAN,OPNET simulation, Entropy,performance indicator

\section{ABSTRACT}

Aiming at the problem that the weight of complex performance index is difficult to make decision in wireless network environment, put forward a weight evaluation method using entropy to determine the indicator weight and construct the weight evaluation model of entropy weight method to evaluate the performance index objectively. With smoothly increasing the node size of wireless workstations, OPNET network simulation software is adopted to record network performance data. After analysis, the entropy information can be used to feedback the contribution degree of performance index information objectively and accurately. The weight decision-making method based on entropy method make reflect the relative importance of network performance index objectively. Moreover, because of its strong adaptability and good flexibility, this method can be widely used in the evaluation of the importance of various network performance indicators.

\section{Introduction}

Wireless local area network (WLAN), as one of the key technologies of network communication system, is widely used in park network, home network, enterprise office network, medical network, etc. With the widespread use of WLAN devices and BYOD/BYOA architecture, WLAN, as a carrier terminal, the application of the link carrier, is playing a more and more important role [1].

Up to now, the performance index of WLAN has not appeared uniform standard, with different network environment, application architecture and business system, the overall performance of WLAN is quite different. Different architecture, environment, application and business constraints must be considered, and the corresponding wireless local area network architecture should be designed. Therefore, it is necessary to put forward the corresponding WLAN performance index analysis method. Compared with the method of coefficient of variation, the paper makes a comprehensive evaluation of the performance of the network system by literature [2]. In the literature, the path performance is weighted average, and the evaluation value is calculated [3]. The evaluation model of network performance is presented based on the global and holistic view of literature [4]. However, the literature above in the process of weight calculation of performance indicators are weighted by the subjective way, which leads to the influence of subjective factors on the evaluation index.

In order to scientifically and reasonably evaluate the performance index of WLAN, avoid using subjective assignment method to bring the weight problem, this paper introduces the entropy of different performance indexes of WLAN law giving weight to establish entropy weight evaluation model, to objectively and accurately reflect the weights in the performance index under WLAN environment. the WLAN performance parameters are obtained through OPNET network simulation, and the WLAN performance evaluation model based on entropy weight method is constructed. Data analysis results show that the method can accurately reflect the degree of information contribution of each WLAN network performance index, and can objectively, accurately and truly reflect the relative importance of different performance indicators in this environment.

\section{CONCEPT OF ENTROPY WEIGHT METHOD}

2.1 Definition of Information Entropy
The concept of "entropy" was proposed by the German physicist Rudolf Clausius in 1850, used to measure the uniformity of any energy distribution in space, the more uniform the distribution of energy, the greater the entropy. C E Shannon in 1948 fused the entropy of the energy level and the degree of information, and introduced it into information theory, producing the information entropy [5]. In information theory, entropy is also called the average amount of information, so as to measure the information. The definition of information entropy is as follows:

Assuming the uncertain random event $x$ produces $n$ results which are $x_{1}, x_{2}, \ldots, x_{n}$ respectively, and independent of each other. The probability of occurrence of each result is $p_{1}, p_{2}, \ldots, p_{n}$.Among, $0 \leq p_{i} \leq 1$, $i=1,2, \ldots, n, \sum_{i=1}^{n} p_{i}=1$. C E Shannon introduced the function $H(x)=-k \sum_{i=1}^{n} p_{i} \ln p_{i}$ to measure the event $x . k$ is a constant greater than 0 , and $H(x)$ is called the information entropy of event $x$.

\section{Entropy weight method}

The concept of entropy is used to assign the weight of index weight, which is called entropy weight method [6]. The entropy weight method is used to measure the information provided by the measured data to make it have a strong objectivity. Entropy method according to different degrees of the value of evaluation objects for an index to reflect the degree of importance. If the difference of the same index value of different evaluation objects is not big, it indicates that the index value plays a very small role in the evaluation system. Entropy weight has specific meaning, not the relative importance coefficient of an index in the evaluation model, but the relative competition intensity coefficient of each evaluation model performance index when the evaluation index value is given in the given evaluation model set. The entropy weight depends on the model being evaluated. From the point of view of information, the entropy weight shows the degree of useful information contributed by this index in this problem [7].

From the above, the smaller the entropy is, the larger the entropy weight is. This indicates that the larger the index information is, the more important the index is. However, the larger the entropy is, the smaller the entropy weight is, which indicates that the index is not important in the 
evaluation model. In this way, it can objectively reflect the importance of an index in the evaluation model.

The introduction of WLAN performance evaluation of the entropy weight method has following advantages: adopting the entropy weight method can exclude the subjective interference model performance evaluation index, and obtain more objective and more realistic evaluation results; by calculating the entropy of each performance index, it can be used to measure the magnitude of the index information, and then guarantee the extent of the original information quantity reflected by the performance index.

\section{WEIGHT EVALUATION MODEL OF ENTROPY WEIGHT METHOD}

\subsection{Constructing Evaluation Matrix}

Set the selected object set as $M=\left(M_{1}, M_{2}, \ldots, M_{n}\right)$ and performance index set is $D=\left(D_{1}, D_{2}, \ldots, D_{n}\right)$. The $D_{j}$ performance index of the evaluated object $M_{i}$ is $x_{i j}(i=1,2, \ldots, m, j=1,2, \ldots, n)$, and the constructed evaluation matrix $X$ is:

$$
X=\left(\begin{array}{ccccc} 
& D_{1} & D_{2} & \cdots & D_{n} \\
M_{1} & x_{11} & x_{12} & \cdots & x_{1 n} \\
M_{2} & x_{21} & x_{22} & \cdots & x_{2 n} \\
\vdots & \vdots & \vdots & & \vdots \\
M_{m} & x_{m 1} & x_{m 2} & \cdots & x_{m n}
\end{array}\right)
$$

\subsection{Standardized Evaluation Matrix}

In order to eliminate the impact of different index attributes on the evaluation of different schemes, or some negative value index evaluation problems, it is necessary to standardize the evaluation matrix $X$ to form a standardized matrix $V=\left[v_{i j}\right\rfloor_{m \times n}$. According to the different characteristics of the index, it can be divided into two categories: one is the benefit type index, the greater the representative of the overall system performance, the better indicators of efficiency; the other is the cost index, which means that the larger the index value is, the worse the overall system performance is.

According to the nature of different indexes, the corresponding standardization forms should be adopted in the process of standardization:

Standardization of efficiency index:

$$
v_{i j}=\frac{x_{i j}-\min x_{j}}{\max x_{j}-\min x_{j}}
$$

Standardization of cost index:

$$
v_{i j}=\frac{\max x_{j}-x_{i j}}{\max x_{j}-\min x_{j}}
$$

After the normalization of $x_{i j}$, obtain the $v_{i j} ; \max x_{j}$ and $\min x_{j}$ are the maximum and minimum values of the $j$ index. It can be obtained that $0 \leq v_{i j} \leq 1$ from the above formula.

\subsection{Characteristic Proportion Calculation of The First $i$ Evaluation Model Under Item $j$}

4.4

For a performance index $j$, the greater the $v_{i j}$ value difference is, the greater the role plays in the evaluation model, that is, the more information the index provides. The reduction of entropy means the increase of information, and entropy can be used to evaluate the amount of information [8].

The characteristic proportion of the first $i$ evaluation model under item $j$ is $p_{i j}$. Then $p_{i j}=\frac{v_{i j}}{\sum_{i=1}^{m} v_{i j}}$

Because of $0 \leq v_{i j} \leq 1$, thus, $0 \leq p_{i j} \leq 1$.

4.5 Determinating the Entropy of the First $j$ Index $e$

$e_{j}=-\frac{1}{\ln m} \sum_{i=1}^{m} p_{i j} \ln p_{i j}$

When $p_{i j}=0$ or 1 ,there is $p_{i j} \ln p_{i j}=0$.

\subsection{Calculating difference coefficient $d$}

According to the entropy formula can be obtained for a performance index $D_{j}$, the smaller the $v_{i j}$ difference is, the bigger the $e_{j}$ is. When the value of a performance index of the evaluation model is all the same, then $e_{j}=e_{\max }=1$. According to the concept of entropy, the greater the difference of an index value, the more information that the index value can reflect. Accordingly, the greater the difference coefficient $d_{j}$, $d_{j}=1-e_{j}$, the more information provided by this performance index and the greater the index weight should be given.

\subsection{Determination of Entropy Weight of Each Performance Index}

$w_{j}=\frac{d_{j}}{\sum_{k=1}^{n} d_{k}}, j=1,2, \cdots, n$

\section{WLAN PERFORMANCE EVALUATION METHOD}

\subsection{Network Performance Evaluation Index Selection}

In the evaluation process of network performance, it is necessary to make meaningful and targeted choices for a number of indicators. For example, in VOIP environment, the time delay index needs to be selected. Connectivity has no practical significance for evaluating the model in VOIP environment, so it is not necessary to consider. Therefore, in the process of index selection of network performance evaluation, the principles of choice should be considered are as follows: comprehensiveness: the selection of indicators is not much, but ensure comprehensive; testability: easy to measure indicators; business nature: performance metrics for different business characteristics [9]. After analysis, this paper selects delay, network load and throughput as WLAN performance evaluation index. The indicators are briefly summarized as follows:

Delay: the time interval of receiving and transmitting data packets in the process of communication. In WLAN environment, excessive delay will bring business on real time service is greatly reduced.

Throughput: the amount of data passing through an interface / channel between units. Throughput is one of the important indexes to measure WLAN network, which reflects the efficiency of network operation in WLAN environment.

Network load: network carrying traffic situation, is a manifestation of network transmission capacity. This index can reflect the network load situation in each WLAN BSS area, and feedback the network transmission capability under the BSS.

In the selected WLAN performance indicators, the longer the delay, the worse the transmission quality and performance; and the larger the throughput and network load, the better the transmission quality and performance. Therefore, in the weight evaluation model of entropy weight, the delay is the cost index, and the throughput and the network load are the benefit indicators.

\subsection{Sample Acquisition of Network Performance Evaluation Index Parameter}

WLAN real network environment, it is easier to obtain its parameters. 
However, the parameter acquisition of the WLAN architecture under the objective, stable and independent application environment is more complex. In general, WLAN environment can carry all kinds of applications, but it is relatively complex to test in a practical environment under different applications of a fixed application or fixed architecture model environment. In this way, it is difficult to obtain the performance index in different states. However, the network simulation method can simulate any changes in the actual situation, and the acquisition of various parameters and performance index sample values can be more intuitive, fast and convenient. Therefore, the OPNET simulation method is adopted in this paper, and obtains several application instances in wireless network environment, and gets the above-mentioned nodes with different nodes under the same conditions [10].

\section{OPNET SIMULATION EXAMPLE ANALYSIS}

\subsection{Method for Obtaining WLAN Performance Index}

Using OPNET simulation software to build WLAN simulation environment to obtain sample parameters. This experiment takes four APs, four BSSs (from 1 to 4), each AP as an area of BSS. The four APs communicate with the server through the switch. Large file transfer service model is adopted between wireless receiving client and server to deploy application. Each client of AP belongs to the BSS of its AP. In order to make the result objective and fair, the size of the wireless receiving client will smoothly increase gradually. Different sizes of nodes form different 10 WLAN scale instances. In order to make the comparison and analysis convenient, different WLAN scene instances have the same parameters except the size of the wireless receiving client. WLAN simulation attribute parameter settings such as Table 1, one of the simulation scene as shown in Figure 1. The simulation time is set to 4 hours, and the throughput, network load and delay value of different scenarios are obtained. The sample parameter values of the WLAN performance index are shown in Table 2.

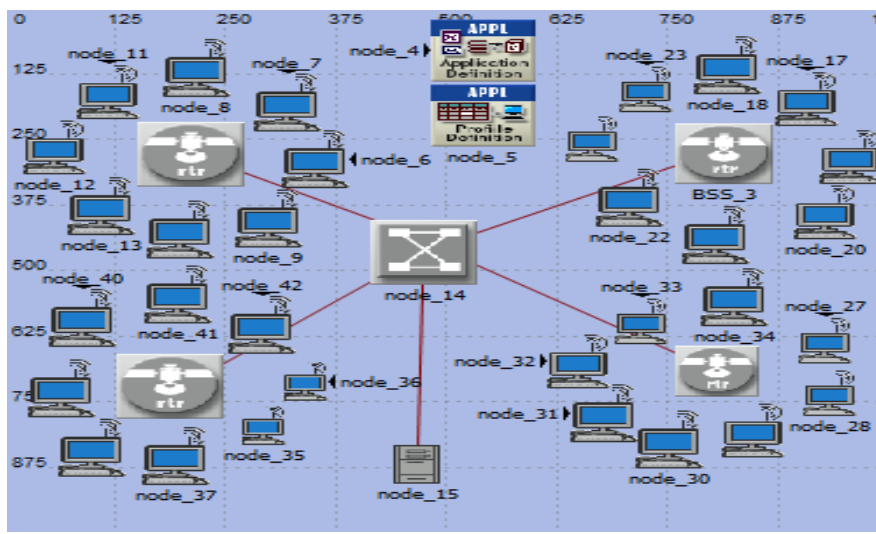

Figure 1: Simulation of WLAN scene based on OPNET

Table 1: Simulation attribute parameter setting of WLAN

\begin{tabular}{|c|c|c|c|c|}
\hline attribute & BSSID & $\begin{array}{c}\text { Physical layer } \\
\text { standard }\end{array}$ & Band & $\begin{array}{c}\text { Transmission } \\
\text { speed }\end{array}$ \\
\hline $\begin{array}{l}\text { parameter } \\
\text { values }\end{array}$ & $1,2,3,4$ & IEEE $802.11 \mathrm{~g}$ & $2.4 \mathrm{Ghz}$ & $54 \mathrm{Mbps}$ \\
\hline
\end{tabular}

Table 2: Statistic values of WLAN performance index

\begin{tabular}{lllllll}
\hline $\begin{array}{l}\text { Node } \\
\text { Numbr } \\
\text { (indivi- } \\
\text { dual) }\end{array}$ & Delay & $\begin{array}{l}\text { BSS_1 } \\
\text { Network } \\
\text { load } \\
\text { (bits/s) }\end{array}$ & $\begin{array}{l}\text { BSS_2 } \\
\text { Network } \\
\text { load } \\
\text { (bits/s) }\end{array}$ & $\begin{array}{l}\text { BSS_3 } \\
\text { Network } \\
\text { load } \\
\text { (bits/s) }\end{array}$ & $\begin{array}{l}\text { BSS_4 } \\
\text { Network } \\
\text { load } \\
\text { (bits/s) }\end{array}$ & $\begin{array}{l}\text { Throughput } \\
\text { (bits/s) }\end{array}$ \\
\hline 10 & 0.000971 & 3443.884 & 3561.307 & 2384.231 & 2472.404 & 11862.81 \\
\hline 17 & 0.000982 & 4738.231 & 5298.009 & 5739.187 & 4356.671 & 20134.24 \\
\hline 26 & 0.000977 & 7916.942 & 7240.08 & 9535.827 & 8771.58 & 33467.54 \\
\hline 32 & 0.000983 & 8506.03 & 9153.84 & 9124.56 & 9271.87 & 36060.91 \\
\hline 41 & 0.003889 & 12008.38 & 12508.91 & 11332.72 & 12773.49 & 48629.42 \\
\hline 48 & 0.004153 & 15541.07 & 14010.90 & 13804.78 & 14452.24 & 57816.03 \\
\hline 56 & 0.004273 & 15629.41 & 16218.57 & 16688.82 & 16336.72 & 64881.84 \\
\hline 62 & 0.004455 & 19721.17 & 18189.56 & 19043.84 & 17484.69 & 74448.52 \\
\hline
\end{tabular}

\begin{tabular}{lllllll}
\hline 68 & 0.004380 & 20014.43 & 19160.44 & 19190.02 & 21286.39 & 79657.44 \\
\hline 77 & 0.004283 & 19662.02 & 23370.92 & 23194.23 & 22448.16 & 88666.73
\end{tabular}

Table 3: WLAN performance index of entropy weight and entropy value

\begin{tabular}{lll}
\hline performance index & Entropy value & Entropy weight \\
\hline Delay & 0.7295 & 0.3348 \\
\hline BSS_1 Network load & 0.8838 & 0.1439 \\
\hline BSS_2 Network load & 0.8826 & 0.1454 \\
\hline BSS_3 Network load & 0.9059 & 0.1165 \\
\hline BSS_4 Network load & 0.8962 & 0.1285 \\
\hline Throughput & 0.8943 & 0.1308 \\
\hline
\end{tabular}

\subsection{Data analysis}

From the statistical parameter values of the performance index samples in Table 2, it indicates that the network delay, the BSS network load and the throughput have little change when the node size is small. With the scale of network wireless workstation node becoming larger, each performance index has also changed greatly. This shows that the operation and status of the network can be reflected by these performance indicators.

Due to the different kinds of network performance indicators, it is difficult to get the magnitude of the network performance index and the relative importance of each performance index from the data sample value of Table 2. Therefore, after obtaining the network performance index sample value, according to the entropy weight evaluation model proposed above, the entropy weight and entropy value of each WLAN performance index are calculated, and the results are shown in Table 3.

The change of WLAN performance index can be reflected by the way of information entropy, and the difference shows that the performance index is sensitive to the change of node scale. So the WLAN performance change can be accurately feedback in this way. The data in Table 3 show that the delay is smaller than the load and throughput of the BSS network. The entropy weight calculated by entropy can be found that in the WLAN environment, when the size of the wireless workstation node becomes larger, the delay weight occupies the dominant position, and has the most comprehensive impact on the performance of WLAN. Compared with BSS_1 and BSS_2 network load and throughput, BSS_3 and BSS_4 network load have little influence on scale increase. Therefore, in the actual engineering design or architecture deployment and selection process, attention should be paid to the impact of delay on the overall performance of WLAN. Besides, in the design of wireless access point architecture of region 1 and 2, except the time delay, the comprehensive performance influence of throughput and network load should be considered. Finally, further consider the impact of throughput performance metrics in this WLAN environment.

\section{CONCLUSION}

For the WLAN performance evaluation process, it is difficult to determine the weights of different indexes, and most of the evaluation of network performance problems under the influence of subjective factors, the performance index weight insufficient. Application of entropy to network comprehensive performance evaluation index and construction of weight evaluation model based on entropy weight method of wireless network performance index, according to the performance index of the network itself, can calculate the weight coefficient objectively, which lay a certain theoretical basis for the wireless network performance index weight problem and effectively avoid the evaluation process of subjective factors. The experimental results show that the information entropy can objectively and accurately feedback the contribution of each performance index in the wireless network environment to the performance index, and the method based on entropy weight evaluation reflects the relative importance of wireless network performance metrics.

\section{ACKNOWLEDGMENTS}

Thanks to the Guangxi Key Laboratory of building new energy and energy conservation for the support of this project (No. 15-J-21-1).

\section{REFERENCES}

[1] Crossler, R.E., Long, J.H., Loraas, T.M., Trinkle, B.S. 2014. Understanding Compliance with BYOD (Bring Your Own Device) Policies Utilizing Protection Motivation Theory: Bridging the Intention Behavior 
Gap[J]. Journal of Information Systems, 28 (1), 209-226.

[2] Huaqiong, Z., Xuewen, T. 2013. Evaluation Model of Network Service Performance Based on Fuzzy Analytic Hierarchy Process [J]. Journal of Computer Applications, 33 (11), 3035 -3038.

[3] Zhengxin, H., Yang, S. 2013. Network Security Situational Assessment Based on Link Performance Analysis [J]. Journal of Computer Applications, 33 (11), 3224-3227.

[4] Fangfang, L., Jing, W., Cunxiang, C. 2013. Service Performance Evaluation Method for Wireless Mesh Network [J]. Application Research of Computers, 30 (2), 551-553.

[5] Zheru, L., Dianhong, Z., Liqing, H. 2014. Information Entropy Analysis of HRV Signals Based on SBF Algorithm [J]. Chinese Journal of Medical Physics, 31 (1), 4652-4655.
[6] Subathra, M.S.P., Suviseshamuthu, E.S., Victoire, T.A.A., A. Christina, H., Amato, U. 2014. A Hybrid with Cross-entropy Method and Sequential Quadratic Programming to Solve Economic Load Dispatch Problem [J] IEEE Systems Journal, 9 (3), 1031-1440.

[7] Yun, L., Min, X., Bo, H., Chaoqing, Z. 2012. Application of TOPSIS Fixed Weight FCE Method in Logistics Supplier Application [J]. Application Research of Computres, 29 (5), 1806-1808.

[8] Sui, Z., Mei, Z., Guotai, C. 2010. Evaluation Model of Science and Technology Based on Entropy Weight Method and Its Empirical Study [J]. Chinese Journal of Management, 7 (1), 34 -42.

[9] Xiaohui, C., Qiliang, L., Junquan, H. 2013. Research on Objective Weighting Method of ZigBee Network Performance Index [J]. Application Research of Computers, 30 (10), 3068-3070.

[10] Braga, A., Araujo, J., Rodrigues, J. 2013. Implementation of a new propagation model for $5.8 \mathrm{GHz}$ systems in OPNET simulator [C]. Antennas and Propagation (EuCAP), 2013 7th European Conference on. IEEE, 954957. 